\title{
Nature of bulges in the Hubble sequence
}

\author{
Eija Laurikainen $^{1}$, H. Salo ${ }^{1}$, R. Buta ${ }^{2}$ and J. H. Knapen ${ }^{3}$ \\ ${ }^{1}$ Division of Astronomy, Dept. of Phys. Sci., Univ. of Oulu, Finland \\ email: eija.laurikainen@oulu.fi \\ ${ }^{2}$ Department of Physics and Astronomy, Univ. of Alabama, USA \\ ${ }^{3}$ Instituto de Astrofisica de Canarias, E-38200, La Laguna, Spain
}

\begin{abstract}
We evaluate the nature of bulges in the Hubble sequence, based on an analysis of 216 disk galaxies of all morphological types (S0-Sd). For this purpose we collect our various analysis made separately for early and late-type galaxies, published in a series of papers. Using the criteria by Kormendy \& Kennicutt we find strong evidence of pseudobulges in all Hubble types, including S0s. However, due to the relatively massive bulges in $\mathrm{S} 0-\mathrm{S} 0 /$ a galaxies it is not probable that the (pseudo)bulges in these galaxies were formed in a similar manner as in spirals. More likely, pseudobulges particularly in strongly barred early-type disk galaxies are suggested to be a combination of star formation and the nearly exponential central components of bars.
\end{abstract}

Keywords. galaxies: structure, galaxies: bulges, galaxies: evolution

\section{Introduction}

The nature of bulges is one of the key issues for evaluating how galaxies form and evolve. Are they mainly structures formed in mergers of disk galaxies, or rather pseudobulges formed by secular evolutionary processes of the disk material? Answering this question has implications also to our understanding of the formative processes of bars and, in case of the early-type disk galaxies, of how black holes form in galaxies.

It has been discussed by Kormendy \& Kennicutt (2004) that prolonged star formation in the central regions of galaxies can explain the masses of bulges in intermediate and late-type spirals, but not in S0-S0/a galaxies. Instead, bulges in early-type disk galaxies are generally assumed to be formed by galaxy mergers in the distant past. However, there is recent evidence that bulges also in many early-type galaxies have characteristics of disks: they have on average fairly exponential surface brightness profiles (Laurikainen et al. 2005, 2006), and some of them have also kinematic evidence of a pseudobulge (Cappellari et al. 2005). An interesting question is how pseudobulges can form in the early-type disk galaxies?

We analyze the nature of (pseudo)bulges using properties of bars and bulges for 216 disk galaxies. The sample consists of spirals from the Ohio State University Bright Spiral Galaxy survey (OSUBSGS, Eskridge et al. 2002,) and early-type disk galaxies from the Near-InfRared S0 galaxy Survey (NIRS0S; Laurikainen et al. 2005, 2006, Buta et al. 2006; www.oulu.fi/astronomy/nirs0s or bama.ua.edu/ rbuta/nirs0s.html; see also Buta et al. in this meeting). For this purpose we collect the various analysis we have previously made for early and late-type disk galaxies using Fourier methods and by applying a 2D multicomponent decomposition code to the surface brightnesses (Laurikainen et al. 2007).

\section{The outlined picture}

(1) Bulges in spiral galaxies of types Sb and later are mainly pseudobulges formed by star formation in the central regions of the disk. 

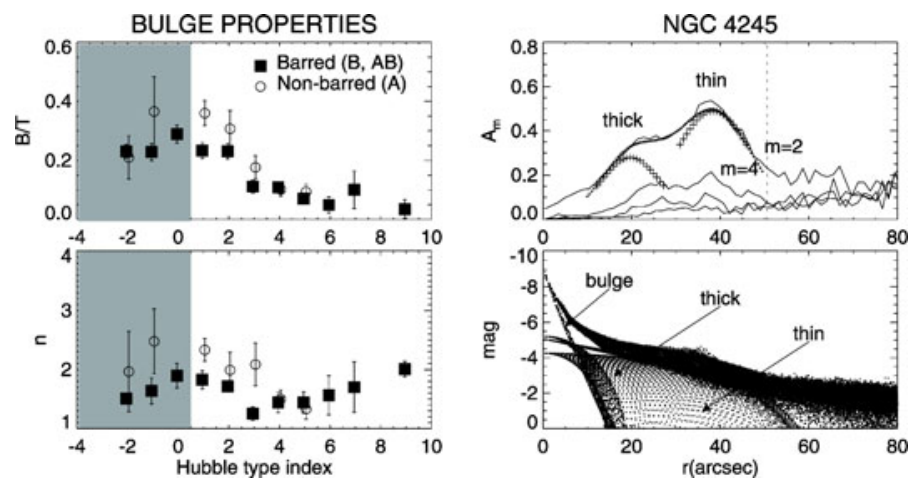

Figure 1. (Left:) bulge-to-total $(B / T)$ flux ratio and Sersic index $n$ as a function of Hubble type index, shown separately for barred and non-barred galaxies. (Right:) $m=2,4,6$ and 8 Fourier amplitude profiles (upper panel) and a multicomponent decomposition (lower panel) for NGC 4245. In the upper panel over-plotted are also Gaussian fits to the $m=2$ component. "Thin" and "thick" refer to the outer and middle components of the bar, whereas "bulge" is a pseudobulge, suggested to be a combination of star formation and the central bar component.

(2) Bulges in SO-SO/a galaxies are also mainly pseudobulges, although classical bulges also appear among these galaxies. For all Hubble types the disk-like nature of the bulge is supported by the small values of the Sersic index ( $n<2$; Fig. 1 , left panels). Also, $56 \%$ of S0-S0/a galaxies have inner rings, disks, or nuclear bars in the region of the bulge.

(3) (Pseudo)bulges in barred and non-barred early-type disk galaxies may have a different interpretation: bulges, particularly in galaxies with strong evolved bars are dominated by the nearly exponential central components of bars (see Fig 1, right panels), which interpretation is consistent with the simulation models by Athanassoula \& Misioritis (2002). However, in non-barred early-type galaxies bulges are more likely classical bulges. In this view it is possible also to understand our finding that $B / T$ flux ratios and $n$-values are smaller in barred than in non-barred early-type galaxies (see Fig. 1, left panels).

(4) Bars in early-type disk galaxies are evolved systems: $90 \%$ of them have flat or intermediate type surface brightness profiles and $40 \%$ have ansae-type morphologies (in contrast to $12 \%$ in spirals). Also, bars are longer and more massive towards the early-type systems. In simulation models this kind of features are identified in evolved bars.

(5) $\mathrm{In} \mathrm{S}^{-}-\mathrm{S}^{0}$ galaxies central disk components may affect the dynamical processes weakening the bars. Consequently bulges that are part of the bar are also weaker. This kind of weakening of bars is predicted by the models by Athanassoula et al. (2005).

\section{References}

Athanassoula, E. \& Misioritis, A. 2002, MNRAS, 330, 35

Athanassoula, E., Lambert, J. C., \& Dehnen, W. 2005, MNRAS, 363, 496

Buta, R., Laurikainen, E., \& Salo, H. 2006, AJ, 132, 1859

Cappellari et al. 2005, astro-ph/073533

Eskridge, P. et al. 2002, ApJS, 143, 73

Kormendy, J., \& Kennicutt, R. C. Jr. 2004, Ann Rev Astr Ap, Vol 42, 603

Laurikainen, E., Salo, H., \& Buta, R. 2005, MNRAS, 362, 1319

Laurikainen, E., Salo, H., Buta, R., Knapen, J., Speltincx, T., \& Block, D. 2006, AJ, 132, 2634

Laurikainen, E., Salo, H., Buta, R., \& Knapen, J. H. 2007, accepted to MNRAS 